\title{
Iterative Fractional Integral Denoising Based on Detection of Gaussian Noise
}

\author{
Yuanxiang Jiang ${ }^{1}$, Rui Yuan ${ }^{1 *}$, Yuqiu Sun ${ }^{1}$, Jinwen Tian ${ }^{2}$ \\ ${ }^{1}$ School of Information and Mathematics, Yangtze University, Jingzhou, Hubei, China. \\ ${ }^{2}$ School of Automation, Huazhong University of Science and Technology, Wuhan, Hubei, China. \\ * Corresponding author. Tel: 15926520876; email: yuanrui@yangtzeu.edu.cn \\ Manuscript submitted December 04, 2017; accepted March 22, 2018. \\ doi: 10.17706/jsw.13.3.155-167
}

\begin{abstract}
In an image with noise, any operation of denoising for a non-noise pixel will change original information. Recent studies show that the denoising algorithms based on noise achieve impressive performance. Meanwhile, because of the characteristics of fractional calculus, the edge information will be retained, and the smooth texture is enhanced while noise is removed. In this paper, an iterative fractional integral denoising algorithm based on noise is proposed. To begin with, we introduce and analyze the noise detection algorithm based on fractional differential gradient and fractional integral denoising from the theoretical point of view. In particular, logical product is made through image of fractional differential gradient to obtain noise position image, thus achieving noise detection. Next, fractional integral denoising algorithms based on tradition and noises are finished. Then, iterative algorithm is used to do multiple searches of noise and integral denoising. In addition, several traditional denoising algorithms and denoising based on noise points are compared to confirm the practicability and feasibility of noise detection algorithm as well as the effectiveness of denoising algorithms based on noise. Finally, different denoising methods are compared to show the characteristic of iterative fractional integral denoising based on noise. By comparing the image visualization and evaluation parameters after processing, it is shown from the experiment results that the method proposed in this paper has good effect of denoising in both subjective and objective aspects.
\end{abstract}

Key words: denoising algorithm, differential gradient, noise detection, fractional integral

\section{Introduction}

With the rapid development of computer technology, the applications of digital image processing have become more and more widely. In the process of image acquisition, conversion and transmission, an image is inevitable corrupted by noise, which not only seriously affects the visual effect of image, but also results in some difficulties for the subsequent segmentation, recognition, understanding and other high-level image processing [1], [2]. Therefore, research on image denoising has become an important component of digital image processing. There are a lot of classic denoising algorithms, such as mean [3], median, order-statistics, lowpass, wiener, and other filtering algorithms, wavelet denoising method [4], denoising based on partial differential equations, fractal theory [5] and fractional integral denoising algorithm [6], [7]. Although these methods can reduce image noises to various degrees, they all have advantages and disadvantages. Mean filter is simple and fast, but it is likely to blur the overall image. Median filter judges noise points from extreme values in neighborhood, which is easy to misjudge details and partial loss of edge information. There is a common disadvantage in traditional denoising algorithms that while removing noise, 
image edge and texture details can usually be lost, leading to blurred vision.

Based on the applications of fractional Grümwald-Letnikov derivative theory to image enhancement, G. Huang et al. [8] leveraged the characteristics that fractional differential and fractional integral were inverse operations, as well as the image enhancement and image denoising, trying to put forward an image denoising model based on fractional Grümwald-Letnikov integral definition, and employing multiple iterations to control the image denoising effect. However, because the fractional integral operator had the lowpass characteristics, multiple iterations could damage the image details at the same time.

The paper written by G. Huang et al. [9] proposed an image denoising algorithm using fractional-order integral with edge compensation, which set a higher order of integral to build a denoising mask in signal-to-noise ratio (SNR) rise, and when SNR began to decline, a relatively lower order of integral was set. Combined with the edge compensation, the image edge and texture information were partially restored.

Liu et al. [10] introduced an adaptive median filtering denoising algorithm based on the detection of noise. Compared with other median filters, the result of tests showed that method based on noise had better ability to denoise and retain details, but this algorithm could only effectively judge the salt and pepper noise or impulse noise. When the gray value of noise was dynamic, this method would not be applicable.

J.R. Hu et al. [11] proposed an innovation denoising method named FIDA, and presented the numerical implementation rules and results of FIDA for digital image. Zhu et al. [12] proposed an adaptive median filter algorithm based on multi-stage noise detection whose key is to detect the types of noise progressively through the spatial correlation of pixels. Lou et al. [13] introduced weight parameters for the approximation process of digital signal and proposed a method combining masks on eight directions and adjusting the value of weight and integral order, achieving to improve the peak signal-to-noise ratio of the image and retain the image detailed texture better at the same time.

In this paper, an algorithm based on the detection of noise is proposed which applied the theory of fractional calculus to process the images with Gaussian noise. Positions of the noise points are obtained by gradient images in different directions to implement fractional order integral denoising based on noise. Because there is certain noise points missed to detect, an iterative algorithm is leveraged to do multiple location searches.

\section{Associated theories}

Fractional Calculus came into existence 300 years ago. It is dedicated to the research of any order differential and integral operators so that it is generally believed that Fractional Calculus is the expansion and extension of integer order calculus [14]. At the same time, because of the genetic and memorial features of Fractional Calculus, it has become favored by many domestic scholars.

\subsection{Definition of Fractional Calculus}

For a function $y=f(t)$ whose argument $t$ is in the range $(\mathrm{a}, \mathrm{b})$, the $v$-order fractional calculus of function $f(t)$ in $(a, b)$ is written as:

$$
{ }_{a} D_{b}^{v} f(t)=D^{v} f(t)
$$

where the order $v$ is fraction, the equation is called fractional differential when $v>0$. When $v<0$, the corresponding called fractional integral. There are three main definitions of fractional calculus: G-L, R-L and Caputo [15].

(1) Grümwald-Letnikov (G-L) Definition of Fractional Calculus

Progression form of fractional derivative is also described as G-L definition. G-L definition of fractional 
calculus derives from the classical operation rules of integer order derivative for continuous function, and fractional order comes into being after deducing its integer order form. The definition of fractional calculus is pretty suitable for signal processing. Since its operation can be converted to convolution form, G-L definition is more practical.

If the function $f(t)$ is continuous and derivable in closed interval [a, b] $(a<b, a \in R, b \in R)$, the first derivative of $f(t)$ is:

$$
f^{\prime}(t)=\lim _{h \rightarrow 0} \frac{f(t+h)-f(t)}{h}
$$

Then, the second derivative of the function can be deduced:

$$
f^{\prime \prime}(t)=\lim _{h \rightarrow 0} \frac{f(t+2 h)-2 f(t+h)+f(t)}{h^{2}}
$$

Similarly, the $n$-order derivative of function, namely $n$-order differential equation is as follows:

$$
f^{n}(t)=\lim _{h \rightarrow 0} \frac{1}{h^{n}} \sum_{m=0}^{n}(-1)^{m}\left(\begin{array}{l}
n \\
m
\end{array}\right) f(t-m h)
$$

G-L definition of fractional differential is derived from integer order $n$ to fraction order $v$ :

$$
{ }_{a}^{G} J_{t}^{v}=\lim _{h \rightarrow 0} h^{v} \sum_{m=0}^{\left.\frac{t-a}{h}\right]}(-1)^{m} \frac{\Gamma(v+1)}{m !(v-m+1) !} f(t-m h)
$$

Where $v>0$, and $\Gamma(n)=\int_{0}^{\infty} e^{-t} t^{n-1} d t=(n-1)$ !. When the order $v<0$, the fractional integral formula in G-L can be defined as:

$$
{ }_{a}^{G} J_{b}^{-v}=\lim _{h \rightarrow 0} h^{v} \sum_{m=0}^{\left[\frac{t-a}{h}\right]} \frac{\Gamma(v+m)}{m ! \Gamma(v)} f(t-m h)
$$

In summary, the fractional calculus of G-L definition can be expressed as:

$$
{ }_{a}^{G} J_{t}^{v}=\lim _{h \rightarrow 0} \frac{1}{h^{v}} \sum_{m=0}^{\left[\frac{t-a}{h}\right]}(-1)^{m}\left(\begin{array}{c}
v \\
m
\end{array}\right) f(t-m h)
$$

(2) Riemann-Liouville (R-L) Definition of Fractional Calculus

R-L definition of fractional differential is also from classical integer order derivative. For R-L definition, $n$-order differential of function or signal is calculated. Then, the $v$-order integral of calculation results is operated. The complete form is expressed as follows:

$$
{ }_{a}^{R} D_{b}^{-v} f(t)=\left\{\begin{array}{l}
\frac{1}{\Gamma(n-v)} \int_{a}^{b} \frac{f(\xi)}{(t-\xi)^{v+1-n}} d(\xi), n-1<v<n \\
\frac{d^{n}}{d t^{n}} f(t), v=n \in N
\end{array}\right.
$$

(3) Caputo Definition of Fractional Calculus

Caputo definition, another way to define fractional calculus, is often used to solve the problems of initial 
boundary value of fractional differential equations. It is also an improved form based on the G-L and widely used in engineering fields. Caputo fractional differential is defined as:

$$
{ }_{a}^{C} J_{b}^{v} f(t)=\frac{1}{\Gamma(v-n)} \int_{a}^{t}(t-\tau) f^{n}(\tau) d \tau, 0 \leq n-1<v<n, n \in N
$$

Integral form is:

$$
{ }_{a}^{C} J_{b}^{v} f(t)=\frac{1}{\Gamma(v)} \int_{a}^{t}(t-\tau) f(\tau) d \tau,-1<v<0
$$

R-L and Caputo definition are all improved from G-L definition. When the order is negative real or positive integers, they are equivalent. Otherwise, they are not. Appearance of R-L definition can simplify the calculation of the fractional derivative. Caputo definition makes the Laplace transform more concise.

\subsection{Noise Detection based on fractional differential gradient}

Because there is a great difference between noise and its adjacent points in pixels, however gray value of edge in some directions is continuous and transitional. Therefore, through the differential gradient in each direction, we can effectively distinguish between noise and image details.

Assuming the center point $\mathrm{O}, C_{8}^{2}=28$ edge directions in all consist of $\mathrm{O}$ and its surrounding eight points A, B, C, D, E, F, G, and H. Figure 1 is a sample view of differential gradient in six directions.

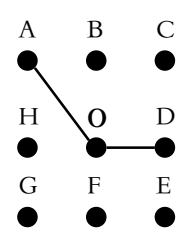

(a)

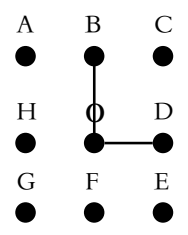

(d)

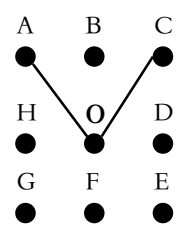

(b)

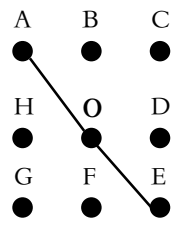

(e)

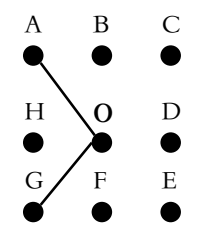

(c)

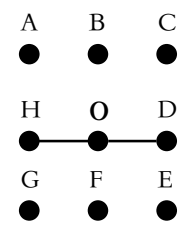

(f)

Fig. 1. Differential gradient in different directions.

The following takes the construction of fractional differential gradient mask in AOD direction for example. The masks in other directions are similar.

According to the calculation formula of fractional differential gradient, the gradient in AOD direction can be shown:

$$
D_{A O-O D}^{v}=D_{A O}^{v}-D_{D O}^{v}=a_{1} I(x-1, y+1)-a_{1} I(x+1, y)+\cdots+a_{n} I(x-n, y+n)-a_{n} I(x+n, y)
$$

where $v$ is the order of differential, the coefficient

$$
a_{1}=-v, a_{2}=\frac{v(v-1)}{2}, a_{3}=\frac{-v(v-1)(v-2)}{6}, a_{4}=\frac{v(v-1)(v-2)(v-3)}{24}, \cdots, a_{n}=(-1)^{n} \frac{\Gamma(v+1)}{n ! \Gamma(v-n+1)}
$$

The mask of differential gradient in AOD direction can obtain through formula (11). 


\begin{tabular}{|c|c|c|c|c|c|c|}
\hline$a_{n}$ & 0 & $\ldots$ & 0 & $\ldots$ & 0 & $-a_{n}$ \\
\hline 0 & $\ddots$ & $\ddots$ & $\vdots$ & $\ddots$ & $\ddots$ & 0 \\
\hline$\vdots$ & $\ddots$ & $a_{1}$ & 0 & $-a_{1}$ & 0 & $\vdots$ \\
\hline 0 & $\ldots$ & 0 & 0 & 0 & $\ldots$ & 0 \\
\hline$\vdots$ & $\ddots$ & $\vdots$ & $\vdots$ & $\vdots$ & $\ddots$ & $\vdots$ \\
\hline 0 & $\ldots$ & 0 & 0 & 0 & $\ldots$ & 0 \\
\hline
\end{tabular}

Fig. 2. The mask of differential gradient in AOD direction.

\subsection{Fractional Integral Denoising}

(1) The theory of denoising by fractional integral

For an arbitrary energy signal $S(x) \in \mathrm{L}^{2}(R)$ [15], while for any operator $D_{v}$ the order $v$ is real and positive, the Fourier transform of $S^{v}(x)$ in frequency domain is:

$$
\left.\left.\left(D_{v} \hat{s}\right) \omega \Rightarrow i \omega{ }^{v} \hat{s}\right) \omega \notin \hat{d}\right) \omega \hat{s} \omega
$$

Exponential form of multiplier $\hat{d}_{v}(\omega)$ in above formula is:

$$
\left\{\begin{array}{l}
\hat{d}_{v}(\omega)=\hat{a}_{v}(\omega) \exp \left[i \theta_{v}(\omega)\right] \\
\hat{a_{v}}(\omega)=|\omega|^{v}, \theta_{v}(\omega)=\frac{v \pi}{2} \operatorname{sgn}(\omega)
\end{array}, v \in R^{+}\right.
$$

From the theory of fractional operators, we can know that $D^{-1}=J \cdot \operatorname{Set} v^{\prime}=-v^{\prime}$, and then the Fourier transform of $S^{v^{\prime}}(x)$ in frequency domain is:

$$
\left(J_{v^{\prime}} \hat{s}\right)(\omega)=(i \omega)^{v^{\prime}} \hat{s}(\omega)=\hat{J}_{v^{\prime}}(\omega) \hat{s}(\omega)
$$

Exponential form of multiplier $\hat{J}_{v^{\prime}}(\omega)$ in formula (14) is:

$$
\left\{\begin{array}{l}
\hat{J_{v^{\prime}}}(\omega)=\hat{a_{v^{\prime}}}(\omega) \exp \left[i \theta_{v^{\prime}}(\omega)\right] \\
\hat{a_{v^{\prime}}}(\omega)=|\omega|^{v^{\prime}}, \theta_{v^{\prime}}(\omega)=\frac{v^{\prime} \pi}{2} \operatorname{sgn}(\omega)
\end{array}, v^{\prime} \in R^{-}\right.
$$

As far as the signal processing, the calculus is equivalent to signal filtering. When $v<0$, the integral operation is equivalent to a lowpass filter. The larger $|v|$ is, the more obvious lowpass characteristics will be.

From the curve of amplitude-frequency response drawn by the relationship $\hat{a}_{v}(\omega)=|\omega|^{v}$, the operator of fractional integral can not only enhance the low frequency signal, attenuate high-frequency signal, but also for the signal which is between low and intermediate frequency, it can reach nonlinear retention [16].

Therefore, it is feasible for calculus to be applied to the image denoising. Moreover, the edge information will be retained, and the smooth texture is enhanced while noise is removed. In particular for the weak edge and texture whose gray level changes little, the operator of fractional integral has a better denoising effect.

(2) Construction of the integral operator template

When $t \in[a, t]$, the duration of signal $f(t)$ is divided into equal parts by the interval $h=1$, then 
$n=\left[\frac{t-a}{h}\right]=[t-a]$. When $v<0$, the approximate differential expression of $v$-order integral of signal $f(t)$ defined by G-L is:

$$
I_{G-L}^{v} f(t) \approx f(t)+(-v) f(t-1)+\frac{(-v)(-v+1)}{2} f(t-2)+\cdots+\frac{\Gamma(-v+1)}{m ! \Gamma(-v-m+1)} f(t-m)
$$

The partial fractional integral in eight directions of a two-dimensional digital image is defined, thus we can generate eight masks. The final mask obtained by superimposing the partial fractional integral in eight directions is shown as Fig.3. The parameters $\xi_{1}, \xi_{2}, \cdots, \xi_{n}$ are coefficients of formula (16).

\begin{tabular}{|c|c|c|c|c|c|c|c|c|}
\hline$\xi_{n}$ & 0 & $\ldots$ & 0 & $\xi_{n}$ & 0 & $\ldots$ & 0 & $\xi_{n}$ \\
\hline 0 & $\ddots$ & $\ddots$ & $\vdots$ & $\vdots$ & $\vdots$ & $\ddots$ & $\ddots$ & 0 \\
\hline$\vdots$ & $\ddots$ & $\xi_{3}$ & 0 & $\xi_{3}$ & 0 & $\xi_{3}$ & $\ddots$ & $\vdots$ \\
\hline 0 & $\ldots$ & 0 & $\xi_{2}$ & $\xi_{2}$ & $\xi_{2}$ & 0 & $\ldots$ & 0 \\
\hline$\xi_{n}$ & $\ldots$ & $\xi_{3}$ & $\xi_{2}$ & $8 \xi_{1}$ & $\xi_{2}$ & $\xi_{3}$ & $\ldots$ & $\xi_{n}$ \\
\hline 0 & $\ldots$ & 0 & $\xi_{2}$ & $\xi_{2}$ & $\xi_{2}$ & 0 & $\ldots$ & 0 \\
\hline$\vdots$ & $\ddots$ & $\xi_{3}$ & 0 & $\xi_{3}$ & 0 & $\xi_{3}$ & $\ddots$ & $\vdots$ \\
\hline 0 & $\ddots$ & $\ddots$ & $\vdots$ & $\vdots$ & $\vdots$ & $\ddots$ & $\ddots$ & 0 \\
\hline$\xi_{n}$ & 0 & $\ldots$ & 0 & $\xi_{n}$ & 0 & $\ldots$ & 0 & $\xi_{n}$ \\
\hline
\end{tabular}

Fig. 3. The superimposing of partial integral mask by 8 directions.

We can acquire these coefficients from the fractional calculus definitions. An integral mask can be constructed as Fig.4. In order to avoid big error while achieving the filter, choose the first three items of G-L definition to construct $5 \times 5$ mask of fractional integral, and ensure isotropic filter. At the same time, to make the overall brightness of the image unchanged after filtering, the filtered images should be normalized.

\begin{tabular}{|c|c|c|c|c|}
\hline$v^{2}+v$ & 0 & $v^{2}+v$ & 0 & $v^{2}+v$ \\
\hline 0 & $v$ & $v$ & $v$ & 0 \\
\hline$v^{2}+v$ & $v$ & 8 & $v$ & $v^{2}+v$ \\
\hline 0 & $v$ & $v$ & $v$ & 0 \\
\hline$v^{2}+v$ & 0 & $v^{2}+v$ & 0 & $v^{2}+v$ \\
\hline
\end{tabular}

Fig. 4. Fractional integral mask of $5 \times 5$.

\section{Results of Image Denoising}

In this section, we add different number of noise to test image. In particular, noise detection algorithm based on fractional differential gradient is introduced and finished. Fractional integral denoising algorithm based on tradition and noises are specially analyzed. In addition, iterative algorithm is leveraged to do multiple searches of noise and integral denoising.

\subsection{Adding and Detecting Noise}

At present, some existing denoising algorithms based on noise mostly aim at removing salt and pepper noise. Gaussian noise is taken into account in this paper. The number of 500,1000,2000,4000 Gaussian noise points whose gray values are between 90 and 170 is randomly added to the original images. 


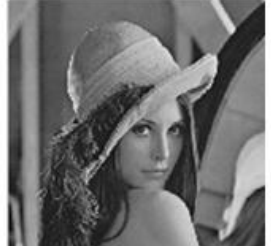

(a)Original

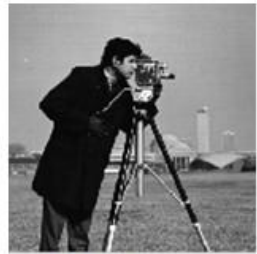

(f) Original

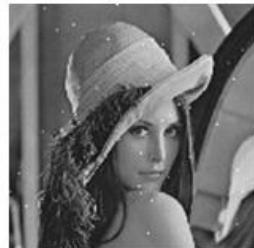

(b) 500

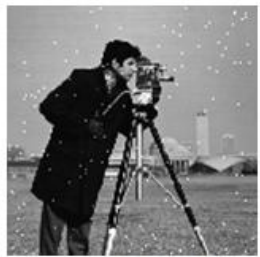

(g) 500

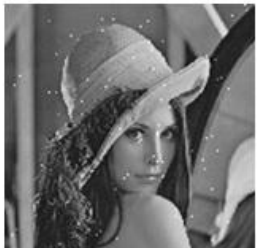

(c) 1000

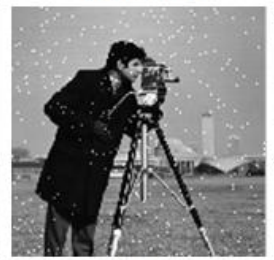

(h) 1000

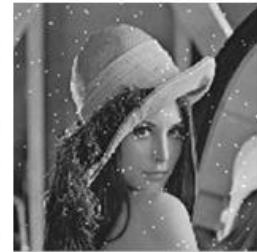

(d) 2000

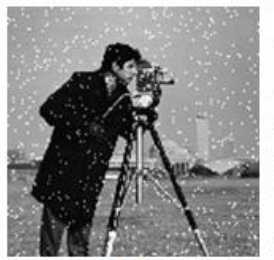

(i) 2000

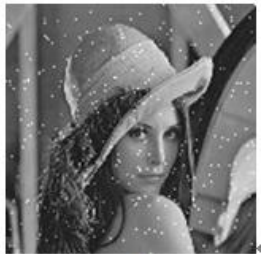

(e) 4000

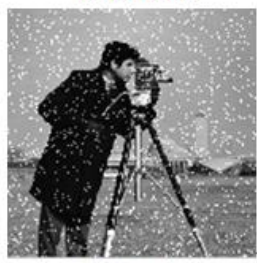

(j) 4000

Fig. 5. Images with different number of noise points.

The application of differential gradient is the key to noise detection. The main process is as follows.

At first, make convolution by noisy image and the direction masks to get direction gradient maps. Then calculate the mean gray value of gradient images and round to nearest, at the same time plus 12 as a threshold value to obtain the gradient detection maps. Finally, through the characteristics that the gradient around noise is discontinuous, but for edge it is continuous and transitional, we can acquire the position of noise and noise image from logical product operation of gradient detection maps.

By comparison of simulation results, it is not difficult to find that direction gradient map from a set of complete direction mask under the fixed initial direction can better eliminate edge information to obtain noise of noisy image.

Hereinafter, seven gradient detection maps based on AO-direction are employed to do logical product. From the features of convolution operation and gradient, we find that there is displacement and overlapping between the position of noise stemming from logical product operation and the actual position, but the rule of variation is substantially the same. Therefore, appropriate inverse transform are leveraged to ascertain the position of noise.

\subsection{Fractional Integral Denoising Based on Noise}

The position image of noise obtained in Section 3.1 and the integral mask constructed in Section 2.3 are used to implement the fractional integral denoising algorithm based on noise, mainly making convolution combined them. For the noise points, after integral operation, the gray values are changed. The rest points keep original values.

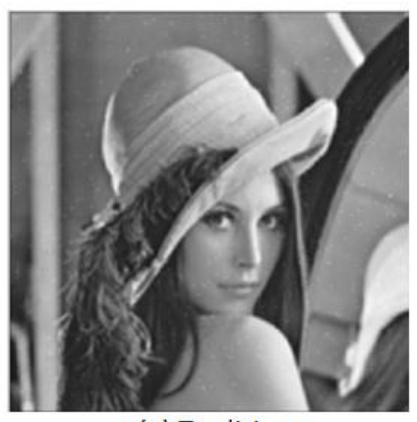

(a) Tradition

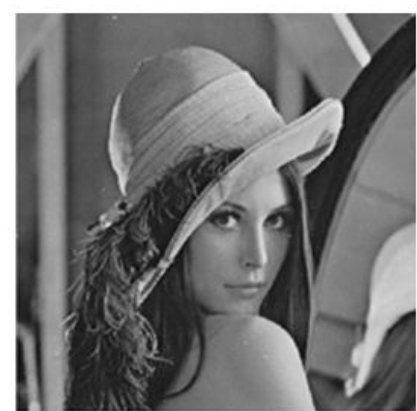

(b) Based on noise

Fig. 6. Fractional integral algorithm based on tradition and noise. 
From Fig. 6, it is not difficult to find that tradition fractional integral algorithm blurs the image, but it based on noise is clearer and greatly improves the visual effect.

In objective aspect, the PSNR (peak signal to noise ratio), MSE (mean square error) and MAE (mean absolute error) are introduced to evaluate and compare various methods.

PSNR is an engineering term that represents the ratio between the maximum possible power of a signal and the power of the corrupting noise that affects its representation. It is defined using the MSE of two images. Define

$$
M S E=\frac{1}{M N} \sum_{i=0}^{M-1} \sum_{j=0}^{N-1}\left[u_{0}(i, j)-u(i, j)\right]^{2}
$$

Then

$$
P S N R=10 \times \log _{10} \max \left(u_{0}, u\right)^{2} / M S E
$$

where $u_{0}$ is the original image without noise, $u$ is the image after denoising; max is the maximum possible pixel value of the image and it is usually equal to 255 in a grayscale image.

The mean absolute error:

$$
M A E=\frac{1}{M N} \sum_{i=1}^{M} \sum_{j=1}^{N}\left|u_{0}(i, j)-u(i, j)\right|
$$

Set different integral orders, and compare various indicators of traditional fractional integral algorithm with method based on noise.

From the Table 1 we can know that for the traditional fractional integral denoising algorithm, with the increase of integral order, PSNR first increases and then decreases; MSE and MAE with an opposite variation trend, which indicates when the order of integral reaches a certain value, there is a conflict between denoising and smoothing. Image texture detail will be weakened more obviously with denoising. However, for the method based on noise, with the increase of the integral order, PSNR gradually increases; MSE and MAE decreases by degrees, and overall the rate of change is quick first and then slow, which indicates that this more stable approach achieves denoising-smoothing to a better harmony.

Table 1. The Values of three Parameters of two Methods in different Orders

\begin{tabular}{lllllll}
\hline \multirow{2}{*}{ Order } & \multicolumn{2}{c}{ Traditional fractional integral } & \multicolumn{3}{c}{ Method based on noise } \\
& PSNR0 & MSE0 & MAE0 & PSNR1 & MSE1 & MAE1 \\
\hline 0.1 & 27.9979 & 0.0016 & 0.0159 & 27.3307 & 0.0018 & 0.0210 \\
0.2 & 29.0452 & 0.0012 & 0.0105 & 28.0696 & 0.0016 & 0.0207 \\
0.5 & 28.2254 & 0.0015 & 0.0273 & 29.7876 & 0.0011 & 0.0199 \\
1.0 & 25.4174 & 0.0029 & 0.0456 & 31.2267 & $7.54 \times 10^{-4}$ & 0.0193 \\
2.0 & 23.1284 & 0.0049 & 0.0621 & 32.0310 & $6.26 \times 10^{-4}$ & 0.0188 \\
5.0 & 22.6371 & 0.0054 & 0.0646 & 32.3684 & $5.79 \times 10^{-4}$ & 0.0186 \\
\hline \hline
\end{tabular}

\subsection{Iterative Fractional Integral Denoising Based on Noise}

Because there are some false detection and missed detection for noise, especially when the MR is high, a bit noise in the processed image will still be clear to poor visual effect.

Based on the topic of iteration, set the time of iteration 1, 10, and 20 respectively, make multiple noise detection and removal in Lena. 


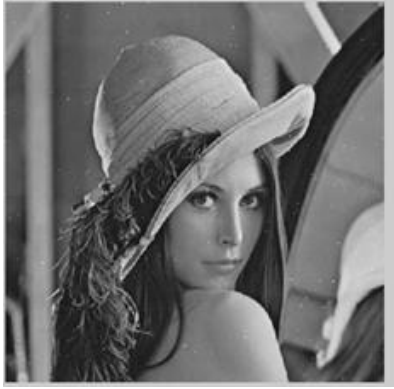

(a) 1

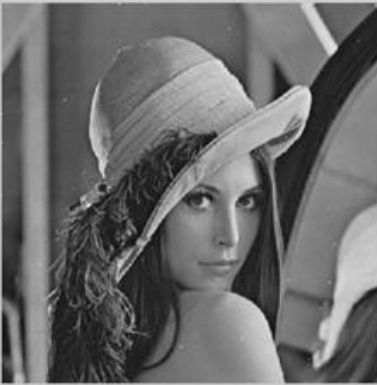

(b) 10

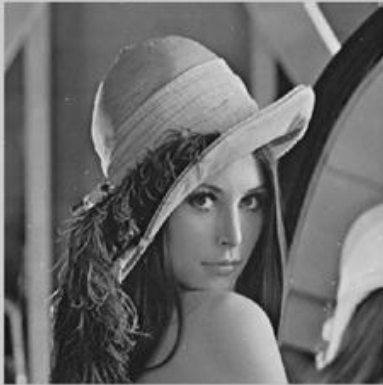

(c) 20

Fig. 7. Images after different iterations.

The tests show that the iterative denoising will reduce the amount of noise points and obviously improve visual quality. However, when the time of iteration reaches a certain number, the improvement is not that significant. What is more, iterative algorithm has high computation cost. Therefore, the appropriate iterative time should be selected. The iteration number is set based on noise level. For higher noise level, we run more times the iteration.

\section{Analysis of Noise Detection and Denoising}

In this section, we present the noise detection results through the number and the ratio of correct detection et.al and compare several traditional denoising algorithms with these methods based on noise points to confirm the practicability and feasibility of noise detection algorithm as well as the effectiveness of denoising algorithms based on noise. Some improved denoising methods are compared to show the characteristic of iterative fractional integral denoising based on noise.

\subsection{Noise Detection}

Test by randomly adding different numbers of Gaussian noise points and measure the effectiveness of detection algorithm by the number and the ratio of correct detection (1 is still corresponding to 1 ), error detection ( 0 turns to 1 ), and missed detection ( 1 turns to 0 ) ( 1 stands for noise and 0 is not noise, the value of the former is for original image and the latter is for result of detection).

Experimental data (see Table2, Table3) show that the ratio of correct detection is all over $90 \%$. At the same time, with the increase of the number of noise points, the $\mathrm{Cr}$ decreases and the $\mathrm{Mn}$ become bigger, but the Er is gradually smaller. Thus, in all, a moderate amount of noise points for the method has better effect on denoising and image enhancement. Moreover, by observing the two tables a strange phenomenon emerges, that is, when the numbers of random noise vary, En are very close. The following work can further study the principles of detection algorithms to reduce Er and increase the accuracy of detection to better realize denoising based on noise.

Table 2. Detection Parameters for different Number of Noise in Lena

\begin{tabular}{ccccccc}
\hline \hline $\mathrm{Nn}$ & $\mathrm{Cn}$ & $\mathrm{Cr}(\%)$ & $\mathrm{En}$ & $\mathrm{Er}(\%)$ & $\mathrm{Mn}$ & $\mathrm{Mr}(\%)$ \\
\hline 500 & 489 & 97.80 & 98 & 19.60 & 11 & 2.20 \\
1000 & 958 & 95.80 & 88 & 8.80 & 42 & 4.20 \\
2000 & 1866 & 93.30 & 85 & 4.25 & 134 & 6.70 \\
4000 & 3650 & 91.25 & 82 & 2.05 & 350 & 8.75 \\
\hline \hline
\end{tabular}

Table 3. Detection Parameters for different Number of Noise in Cameraman

\begin{tabular}{lllllll}
$\mathrm{Nn}$ & $\mathrm{Cn}$ & $\mathrm{Cr}(\%)$ & $\mathrm{En}$ & $\mathrm{Er}(\%)$ & $\mathrm{Mn}$ & $\mathrm{Mr}(\%)$ \\
\hline
\end{tabular}




\begin{tabular}{ccccccc}
\hline 500 & 484 & 96.80 & 184 & 36.80 & 16 & 3.20 \\
1000 & 959 & 95.90 & 183 & 18.30 & 41 & 4.10 \\
2000 & 1885 & 94.25 & 171 & 8.55 & 115 & 5.75 \\
4000 & 3672 & 91.80 & 156 & 3.90 & 328 & 8.20 \\
\hline
\end{tabular}

*Nn, total number of noise; $\mathrm{Cn}$, the number of correct detection; $\mathrm{Cr}$, the ratio of $\mathrm{Cn}$ and $\mathrm{Nn}$; $\mathrm{En}$, the number of error detection; Er, the ratio of En and $\mathrm{Nn}$; Mn, the number of missed detection; $\mathrm{Mr}$, the ratio of $\mathrm{Mn}$ and $\mathrm{Nn}$

\subsection{Comparison with Traditional Denoising Algorithms}

There are many traditional denoising algorithms which can denoise to some extent, but they commonly have the problems of losing and blurring image details. The effects of traditional mean filter, median filter, fractional integral denoising and these methods based on noise are presented in Fig.8 and Fig.9.

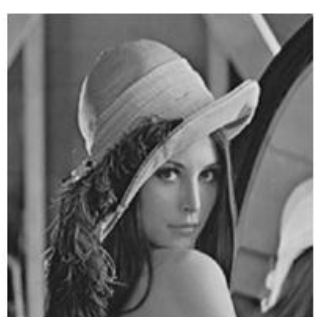

(a) Original

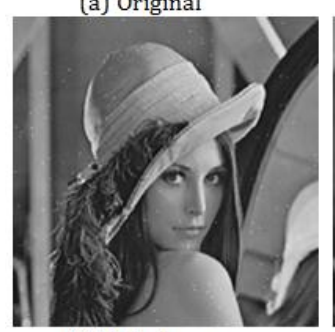

(e)Noisy image

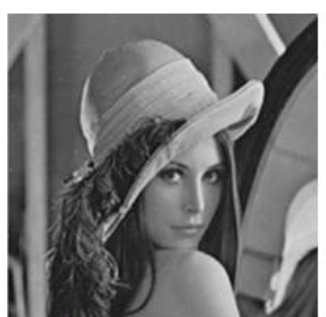

(b) Traditional mean

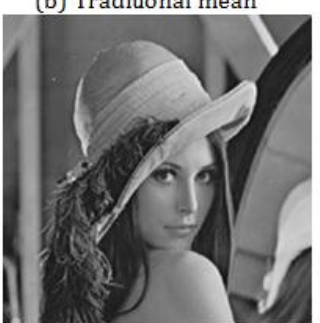

(f)Mean based noise

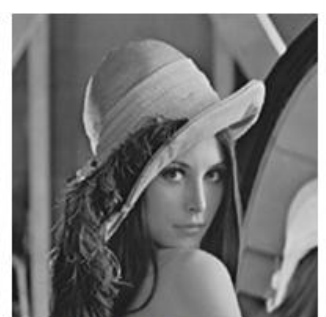

(c) Traditional median

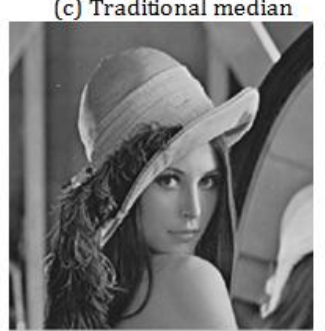

(g) Median based noise (h) Fractional integral based noise

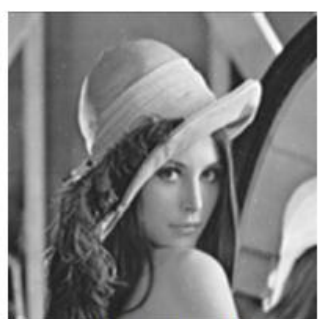

(d) Fractional integral

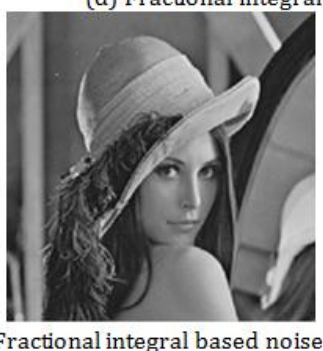

Fig. 8. Traditional denoising algorithms and these methods based on noise for Lena.

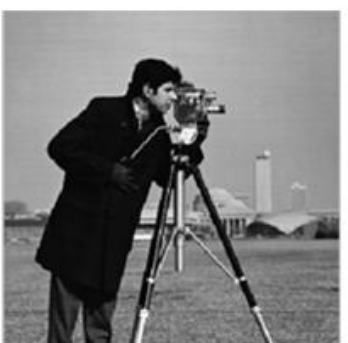

(a) Original

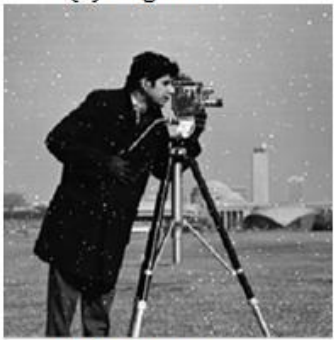

(e)Noisy image

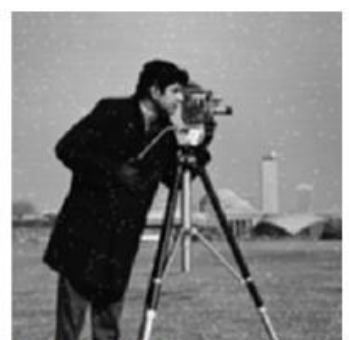

(b) Traditional mean

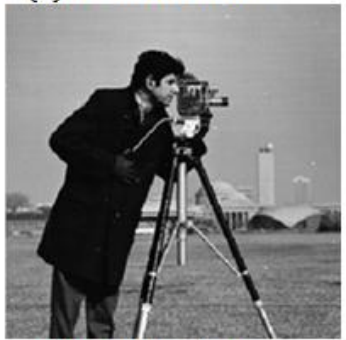

(f)Mean based noise

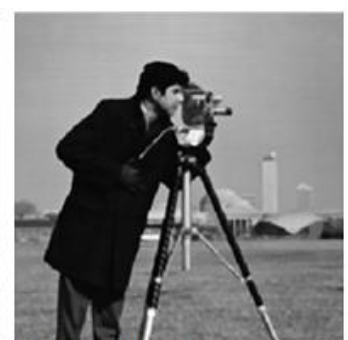

(c) Traditional median

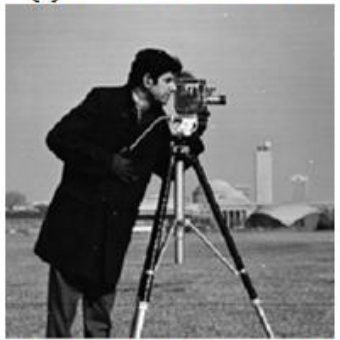

(g) Median based noise

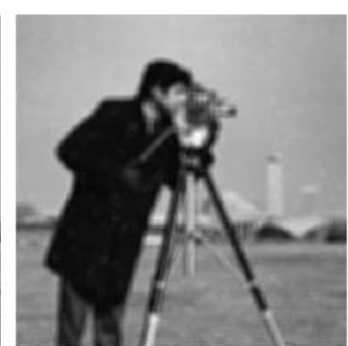

(d) Fractional integral

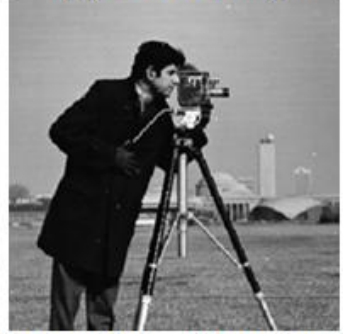

(h) Fractional integral based noise

Fig. 9. Traditional denoising algorithms and these methods based on noise for Cameraman.

By contrast, in subjective or visual aspect, the images after denoising through mean filtering and fractional calculus algorithms become significant blurry. At the same time, noise points are just reduced in 
gray value, and still clearly visible. The algorithms based on noise greatly improve performance on fuzzy extent. However, because there are some noise points missed to detect, nothing to be done for them so that these noise points are particularly significant, but little impact on overall recognition of the image.

Since the characteristics of median filter, the algorithm based on noise does not have obvious superiority over the traditional method in visual.

Table 4. Various Parameters of different Methods in LENA

\begin{tabular}{lcccc}
\hline \hline Methods & & Mean filter & Median filter & Fractional integral \\
\hline \multirow{2}{*}{ Traditional } & PSNR0 & 28.2221 & 22.7358 & 22.8317 \\
methods & MSE0 & 0.0015 & 0.0053 & 0.0052 \\
& MAE0 & 0.0308 & 0.0709 & 0.0633 \\
\hline \multirow{2}{*}{ Methods based } & PSNR1 & 33.3808 & 33.3323 & 32.2157 \\
on noise & MSE1 & $4.59 \times 10^{-4}$ & $5.61 \mathrm{e} \times 10^{-4}$ & $6.00 \times 10^{-4}$ \\
& MAE1 & 0.0181 & 0.0183 & 0.0186 \\
\hline \hline
\end{tabular}

Table 5. Various Parameters of different Methods in Cameraman

\begin{tabular}{lcccc}
\hline \hline Methods & & Mean filter & Median filter & Fractional integral \\
\hline \multirow{2}{*}{ Traditional } & PSNR0 & 25.4018 & 26.1734 & 20.9162 \\
methods & MSE0 & 0.0029 & 0.0021 & 0.0081 \\
& MAE0 & 0.0286 & 0.0303 & 0.0555 \\
\hline \multirow{2}{*}{ Methods based } & PSNR1 & 34.1847 & 33.8426 & 32.9045 \\
on noise & MSE1 & $3.81 \times 10^{-4}$ & $4.13 \times 10^{-4}$ & $5.12 \times 10^{-4}$ \\
& MAE1 & 0.0048 & 0.0046 & 0.0050 \\
\hline \hline
\end{tabular}

In objective aspect, by comparing various parameters (see Table.4 and Table.5), it is not difficult to find that for basic denoising methods, the algorithms based on noise have greater PSNR as well as smaller MSE and MAE than traditional methods. The various values show the practicability and feasibility of noise detection algorithm based on fractional differential gradient.

\subsection{Comparison with Improved Denoising Algorithms}

To further observe the capability of iterative fractional integral algorithm based on noise (IFIN) in the paper, from the view of objective quantitative analysis, PSNR is taken to compare with some other denoising methods, including Fractional Integral Denoising Algorithm (FIDA) in [11], Adaptive Median Filter Algorithm (AMFA) in [12], and Weighted Fractional Integral Algorithm (WFIA) in [13]. The data of the comparison methods are obtained directly from the original authors.

Table 6. PSNR(db) of Several Methods with different Noise Level

\begin{tabular}{cccccc}
\hline \hline Method & Sigma & FIDA & AMFA & WFIA & IFIN \\
\hline \multirow{4}{*}{ Lena } & 5 & 34.860 & 34.951 & 35.272 & 33.694 \\
& 10 & 31.655 & 31.919 & 32.163 & 32.518 \\
& 15 & 28.713 & 30.103 & 30.780 & 31.017 \\
\hline \multirow{4}{*}{ Peppers } & 5 & 34.253 & 34.898 & 34.971 & 33.031 \\
& 10 & 30.630 & 31.563 & 31.708 & 31.953 \\
& 15 & 28.470 & 29.230 & 29.435 & 29.812 \\
\hline \hline
\end{tabular}

From the Table 6 we know that when the sigma of noise is 5, the IFIN has smaller PSNR than the other 
three methods. However, when the sigma of noise is 10 and 15, the PSNR of the IFIN is highest. The experimental data manifest that the iterative fractional integral algorithm based on noise has the general denoising effect for small density noise image, but when the number of noise is large, it is apparently superior to other methods.

\section{Conclusion}

In recent years, the fractional calculus theory has been widely applied to digital image processing and achieved good simulation results. Especially in applications of denoising, since the integral operation corresponds to a set of low-pass filter, denoising algorithm based on fractional integral can better coordinate contradiction between denoising and retaining texture details.

However, any operation of denoising will change the original information for non-noise pixel, so processing only for the noise points will get better denoising performance.

In this paper, because the gradient around noise is transitional and that of edge is continuous, firstly the noise position is calculated through map of fractional differential gradient of noisy images. Then fractional integral denoising based on noise points is operated. Finally, iterative algorithm is used to do multiple searches of noise and integral denoising.

Simulation results show that method proposed in this paper has great denoising effect both in subjective and objective aspects. However, it is still much space for improvement. The future research needs: First, improving detection algorithm based on differential gradient and reducing the ratio of missed detection. Especially when the density of noise is large, how to reduce noise and to control the balance of the ratio of missed detection and the ratio of error detection. Second, paying more attention to the discipline of integral order in the algorithm and trying to set it through the local or global features in order to achieve adaptive denoising. How to combine the method with some other classic denoising algorithm to better coordinate contradiction between denoising and retaining texture details is also an approach.

\section{Acknowledgments}

This work is partially supported by the National Natural Science Foundation of China (11571041), the Natural Science Foundation of Hubei Province (2013CFA053).

\section{References}

[1] Li, B., \& Xie, W. (2016). Image denoising and enhancement based on adaptive fractional calculus of small probability strategy. Neurocomputing, 175, 704-714.

[2] Landi, G., \& Lohp, E. (2012). An efficient method for nonnegative constrained total variation-based denoising of medical images corrupted by Poisson noise. Computer. Med. Imaging Graph, 36(1), 38-46.

[3] Coupe, P., Yger, P., \& Prima, S. (2008). An optimized block wisen on local means denoising filter for 3-D magnetic resonance images. IEEE Trans. Med. Imaging, 27, 425-441.

[4] China, R. B., \& Madhavi, L. M. (2010). A combination of wavelet and fractal image denoising technique. Int. J. Electronic.Eng, 2(2), 259-264.

[5] Ghazel, M., \& E. R. Vrscav. (2001). Fractal image denoising. IEEETrans.ImageProcess, 12(12), 1560-1578.

[6] Lu, J. (2015). Image denoising based on fractional integral. Electronic Technology and Software Engineering, 21,106-164.

[7] Yang, Z. Z., Zhou, J. L., \& Lang, F. N. (2014). Noise detection and image denoising based on fractional calculus. Journal of Image and Graphics, 10, 1418-1429.

[8] Huang, G., Pu, Y. F., Chen, Q. L., \& Zhou. J. L. (2011). Research on image denoising based on fractional order integral. Systems Engineering and Electronics, 4, 925-932.

[9] Huang, G., Chen, Q. L., \& Xu, L. (2014). Image denoising algorithm using fractional-order integral with 
edge compensation. Journal of Computer Applications, 10, 2957-2962.

[10] Liu. M. (2011). Dynamic window-based adaptive median filter algorithm. Journal of Computer Applications, 02, 390-392.

[11] Hu, J. R., Pu, Y. F., \& Zhou, J. L. (2012). Fractional integral denoising algorithm. Journal of the University of Electronic Science and Technology of China, 41(5), 706-711.

[12] Zhu, W., Han, J. F., Chen, P., \& Zhao, B. (2013). Adaptive median filter algorithm based on multi-stage noise detection. Opto-Electronic Engineering, 40(10), 63-69.

[13] Lou, L. T., Fang, Z. C., Wei, X. Y., \& Wu, G. L. (2016). Image denoising method for weighted fractional integral. Journal of South-Central University for Nationalities (Nat. Sci. Edition), 35(03), 146-150.

[14] He, N., Wang, J. B., \& Zhang, L. L. (2015). An improved fractional order differentiation model for image denoising. Signal Process, 112, 180-188.

[15] Huang, G., Xu, L., \& Chen, Q. L. (2013). Image denoising based on Riemann-Liouville fractional integral. Journal of Computer Applications, 33( 1), 35-39.

[16] Ma, Y., Yu, T., Zhao, J. L. \& Li, X. H. (2014). Improved image denoising algorithm based on adaptive fractional integral. Video Engineering, 19, 36-40.

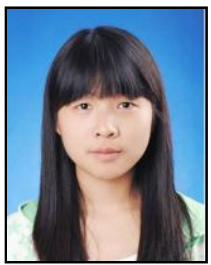

Yuanxiang Jiang was born in Hubei province of China, in 1993, is a master student in the College of Information and Mathematics of Yangtze University. She completed her undergraduate studies in information and mathematical sciences at Yangtze University in 2015. Her current research is about digital image processing. Her research interests include image restoration, target detection and recognition.

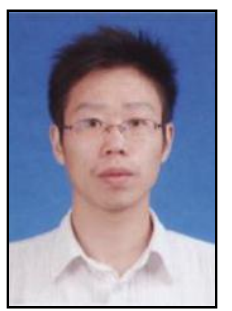

Rui Yuan was born in Hubei province of China, in 1987, is a lecturer and teaches in Yangtze University since 2017. He received his Ph. D. in Yangtze University in 2017. His research interests include optimization theory and its application, image processing and mathematical application in petroleum and other industry.

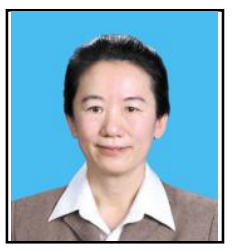

Yuqiu Sun was born in Heilongjiang province of China in 1968, is a professor and teach in Yangtze University since 2003. She received her Ph. D. in Huazhong University of Science and Technology in 2006. She has also served as reviewers of several academic journals. Her research interests include image fusion, wavelet transform theory and its application, image data compression, target detection and recognition.

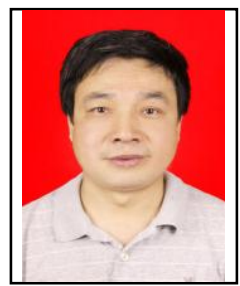

Jinhua Tian was born in 1962, is a professor and teaches in Huazhong University of Science and Technology since 1998. He completed his undergraduate studies in mathematic in school of Daqing Petroleum. He received his master's degree in Huazhong University of Science and Technology in 1994, and received his Ph. D. in 1998. He is senior member of China Electronics Society and has served as reviewers of many academic journals. His research interests include remote sensing image information processing, streaming media technology and its application, wavelet transform theory and its application, image data compression, target detection and recognition, enhanced reality and computer software simulation. 